Notre Dame Journal of Formal Logic

Volume 37, Number 3, Summer 1996

\title{
Classical and Intuitionistic Models of Arithmetic
}

\author{
KAI F. WEHMEIER
}

\begin{abstract}
Given a classical theory T, a Kripke structure $\mathbf{K}=\left(K, \leq,\left(A_{\alpha}\right)_{\alpha \in K}\right)$ is called T-normal (or locally T) if for each $\alpha \in K, A_{\alpha}$ is a classical model of T. It has been known for some time now, thanks to van Dalen, Mulder, Krabbe, and Visser, that Kripke models of HA over finite frames $(K, \leq)$ are locally PA. They also proved that models of HA over the frame $(\omega, \leq)$ contain infinitely many Peano nodes. We will show that such models are in fact PA-normal, that is, they consist entirely of Peano nodes. These results are then applied to a somewhat larger class of frames. We close with some general considerations on properties of non-Peano nodes in arbitrary models of $\mathbf{H A}$.
\end{abstract}

1 Preliminaries A Kripke structure for a language $\mathrm{L}$ is a triple $\mathbf{K}=\left(K, \leq,\left(A_{\alpha}\right)_{\alpha \in K}\right)$ such that $(K, \leq)$ is a (nonempty) partial order (called the frame of $\mathbf{K})$ and for each $\alpha \in K, A_{\alpha}$ is a classical L-structure $A_{\alpha}=\left(A_{\alpha},=_{\alpha},\left(R_{\alpha}\right)_{R \in L},\left(f_{\alpha}\right)_{f \in L}\right)$ (not necessarily normal, that is, $={ }_{\alpha}$ need not be true equality on $A_{\alpha}$ ), with the proviso that the following monotonicity conditions be fulfilled. Whenever $\alpha \leq \beta$, then

1. $A_{\alpha}$ is a subset of $A_{\beta}$;

2. for every relation symbol $R$ of $\mathrm{L}$ (including equality $=$ ), $R_{\alpha} \subseteq R_{\beta}$;

3. for every $n$-ary function symbol $f$ of $\mathrm{L}, f_{\alpha}$ is $f_{\beta}$ restricted to $A_{\alpha}{ }^{n}$.

Throughout this paper, $\mathrm{L}$ will be some suitable version of the arithmetical language with or without symbols for all primitive recursive functions. Forcing, $\Vdash$, is defined as usual. We are treating $\perp$ as a basic connective (so that $\perp$ counts as an atomic formula); negation is defined as $\neg \psi: \equiv \psi \rightarrow \perp$.

Since in HA atomic formulas are decidable, we assume without loss of generality for $\mathbf{K} \models \mathbf{H A}$ that every $A_{\alpha}$ is a normal structure (i.e., ${ }_{\alpha}$ is true equality on $A_{\alpha}$ ) and that for $\alpha, \beta \in K$, if $\alpha \leq \beta$, then $A_{\alpha} \subseteq A_{\beta}\left(A_{\alpha}\right.$ is a substructure of $A_{\beta}$ ) (cf. Markovic [3], Smorynski [4]). $\mathrm{L}_{\alpha}$ is $\mathrm{L}\left(A_{\alpha}\right)$, that is, $\mathrm{L}$ plus constant symbols a for each element $a \in A_{\alpha}$. We often write ' $\alpha \models \varphi$ ' instead of ' $A_{\alpha} \models \varphi$ ', meaning that $A_{\alpha}$ classically satisfies $\varphi$, whereas ' $\alpha \Vdash \varphi$ ' means that $\varphi$ is forced at $\alpha$ in $\mathbf{K}$.

Received November 8, 1995; revised June 25, 1996 
Decidability of atomic formulas in $\mathbf{H A}$ also entails (cf. [3) that for any $\mathbf{K} \models$ HA, every $\alpha \in K$ and every $\Delta_{0}$-sentence $\varphi \in L_{\alpha}$,

$$
\alpha \Vdash \varphi \Longleftrightarrow \alpha \models \varphi \text {. }
$$

A node $\alpha$ in some Kripke structure $\mathbf{K}$ is called classical if $\alpha$ forces every $\mathrm{L}_{\alpha}$-sentence of the form $\forall x_{1} \ldots x_{n}(\varphi \vee \neg \varphi)$. Note that in any Kripke structure, terminal nodes are classical. The following lemma gives some other characterizations of classical nodes.

Lemma 1.1 Let $\mathbf{K}$ be any Kripke structure with the property that $\alpha \leq \beta$ implies that $A_{\alpha}$ is a substructure of $A_{\beta}$, and suppose $\alpha \in K$. The following are equivalent.

1. $\alpha$ is a classical node.

2. $\alpha$ forces every $L$-sentence of the form $\forall x_{1} \ldots x_{n}(\varphi \vee \neg \varphi)$.

3. For every $L_{\alpha}$-sentence $\varphi, \alpha \Vdash \varphi \Longleftrightarrow \alpha \models \varphi$.

4. Whenever $\alpha \leq \beta \leq \gamma, A_{\beta} \prec A_{\gamma}$.

Proof: The equivalence of the first three conditions is well known and was already mentioned in [5]. It remains to show that condition 4 is equivalent to the others. First suppose that 1-3 hold. By 2 and the persistence of forcing, every $\beta \geq \alpha$ is also classical. Let $\alpha \leq \beta \leq \gamma$ and let $\varphi$ be an $\mathrm{L}_{\beta}$-sentence. First assume that $A_{\beta}=\varphi$. Since $\beta$ is classical, $\beta \Vdash \varphi$ and hence $\gamma \Vdash \varphi$. But $\gamma$ is also classical and thus $A_{\gamma} \models \varphi$. Now assume that $A_{\gamma} \models \varphi$. Since $\gamma$ is classical, $\gamma \Vdash \varphi$. Now $\beta$ is classical and $\beta \nVdash \neg \varphi$ (since $\beta \leq \gamma$ and $\gamma \Vdash \varphi$ ), so $\beta \Vdash \varphi$ and $A_{\beta} \models \varphi$.

Now suppose that 4 obtains. We will prove by induction on $\varphi$ that for each $\beta \geq \alpha$ and each $\mathrm{L}_{\beta}$-sentence $\varphi, \beta \Vdash \varphi \Longleftrightarrow \beta \models \varphi$. If $\varphi$ is atomic, our claim follows by definition. The cases of conjunction, disjunction and existential quantification follow easily from the induction hypothesis. Let us consider the case of implication, say $\varphi$ is of the form $\psi \rightarrow \chi$. First suppose $\beta \Vdash \psi \rightarrow \chi$ and $\beta \models \psi$. By the induction hypothesis, $\beta \Vdash \psi$ and hence $\beta \Vdash \chi$. By the induction hypothesis again, $\beta \models \chi$.

Now suppose that $\beta \nVdash \psi \rightarrow \chi$. Then, for some $\gamma \geq \beta, \gamma \Vdash \psi$ and $\gamma \nVdash \chi$. Then clearly $\beta \nVdash \chi$ and thus, by the induction hypothesis, $\beta \not \chi$. Now since $\gamma \Vdash \psi$, by the i.h. $\gamma \models \psi$ and, since $A_{\beta} \prec A_{\gamma}, \beta \models \psi$. The case of universal quantification can be treated analogously.

Given L-formulas $\varphi$ and $\rho$, the Friedman translation of $\varphi$ by $\rho$, denoted $\varphi^{\rho}$, is obtained from $\varphi$ by replacing each atomic subformula $\mathrm{P}$ in $\varphi$ by $\mathrm{P} \vee \rho$ (where it is understood that no variable occurring free in $\rho$ is bound in $\varphi$ ). Some properties of the Friedman translation are the following.

1. $\rho \rightarrow \varphi^{\rho}$ is provable in intuitionistic logic.

2. Classically, $\varphi^{\rho} \leftrightarrow \varphi \vee \rho$.

3. $\mathbf{H A} \vdash \varphi \Rightarrow \mathbf{H A} \vdash \varphi^{\rho}$.

A formula $\varphi$ is semipositive (cf. Buss [1]) if, whenever $\psi \rightarrow \chi$ is a subformula of $\varphi$, $\psi$ is atomic. In particular, only atomic formulas can occur negated (since negation is defined in terms of implication and falsum). Note that classically, every formula is equivalent to a semipositive one (simply eliminate subformulas $\psi \rightarrow \chi$ in favor of $\neg \psi \vee \chi$ and put the resulting formula in negation normal form). Semipositive sentences have the following property. 
Lemma 1.2 Let $\mathbf{K}$ be any Kripke structure, $\alpha \in K$ and $\varphi \in L_{\alpha}$ a semipositive sentence. If $\alpha \Vdash \varphi$, then also $\alpha \models \varphi$.

Proof: By induction on $\varphi$, simultaneously for all $\alpha \in K$. The atomic case is trivial; so are (using the induction hypothesis) the cases of conjunction, disjunction and both quantifiers. It remains to check the case of implication. Suppose $\alpha \Vdash \psi \rightarrow \chi$, where $\psi \rightarrow \chi$ is semipositive. Then $\psi$ is atomic. Suppose further that $\alpha \models \psi$. Since $\psi$ is atomic, $\alpha \Vdash \psi$ and hence, since $\alpha \Vdash \psi \rightarrow \chi, \alpha \Vdash \chi$. $\chi$ is semipositive, so by the induction hypothesis, $\alpha=\chi$.

Note that Lemma 1.2 remains true if we relax the condition in the definition of semipositivity by allowing $\Delta_{0}$-antecedents, provided that in $\mathbf{K}, \Delta_{0}$-formulas are decidable.

2 Introduction Given any Kripke structure $\mathbf{K}$ and $\alpha \in K$, it is tempting to ask which sentences are classically valid in the structure $A_{\alpha}$, as opposed to those sentences intuitionistically true (forced) at $\alpha$.

In the case of Kripke models of Heyting Arithmetic, it appears to be a natural conjecture that every node should be a classical model of Peano Arithmetic. Pressed to substantiate this conjecture, however, one finds little obvious evidence. Moreover, another conjecture that may seem equally plausible on first sight, viz. that PA-normal Kripke structures should always be models of HA, turns out to be false (cf. [1]).

The results recapitulated and presented in this paper, however, may be counted as more or less convincing evidence that our conjecture (of HA-models being locally PA) is, at the very least, a useful working hypothesis. We will proceed by presenting these results in, as we feel, an order of increasing convincing power.

\section{Known Results on Arbitrary Models of HA}

Theorem 3.1 If $\mathbf{K} \models \mathbf{H A}$ and $\alpha \in K$, then $A_{\alpha}=I \Delta_{0}+T h_{\Pi_{2}}$ (PA).

Proof: $\quad A_{\alpha} \models I \Delta_{0}$ : Let $\varphi(x)$ be a $\Delta_{0}$-formula of $\mathrm{L}_{\alpha}$ and suppose that $\alpha \models \varphi(0) \wedge$ $\forall x(\varphi(x) \rightarrow \varphi(S x))$. Assume by way of contradiction that $\alpha \not \forall \forall x \varphi(x)$, say $\alpha \not \models \varphi(b)$ with $b \in A_{\alpha}$. Then $\alpha \nVdash \varphi(b)$ and thus $\alpha \Vdash \neg \varphi(b)$ which entails $\alpha \Vdash \exists x \neg \varphi(x)$. Now the least number principle is provable in $\mathbf{H A}$ for $\Delta_{0}$-formulas and so $\alpha \Vdash \exists x(\neg \varphi(x) \wedge$ $\forall y<x \varphi(y)$, i.e., for some $a \in A_{\alpha}, \alpha \Vdash \neg \varphi(a) \wedge \forall y<a \varphi(y)$. Now $a \neq 0$ since $\alpha \models \varphi(0)$ and thus $\alpha \Vdash \varphi(0)$. So $a$ is a successor, say $a=S c$. Then $\alpha \Vdash \varphi(c)$, so $\alpha \models \varphi(c)$ which in turn implies $\alpha \models \varphi(S c)$ (by validity of the induction step in $A_{\alpha}$ ) and thus $\alpha \Vdash \varphi(S c)$, a contradiction. (This result was first published in [3].)

$A_{\alpha} \models \mathrm{Th}_{\Pi_{2}}(\mathbf{P A})$ : Note that PA is $\Pi_{2}$-conservative over HA. So if PA $\vdash$ $\forall x \exists y \varphi(x, y)$ with $\varphi \in \Delta_{0}$, then $\mathbf{H A} \vdash \forall x \exists y \varphi(x, y)$ and $\alpha \Vdash \forall x \exists y \varphi(x, y)$. In particular, for all $a \in A_{\alpha}, \alpha \Vdash \exists y \varphi(a, y)$, i.e., for each $a \in A_{\alpha}$ there is $b \in A_{\alpha}$ such that $\alpha \Vdash \varphi(a, b)$, i.e., $\alpha \models \varphi(a, b)$.

Remark 3.2 By results of Kaye [2], the theorem does not even imply $A_{\alpha} \models \mathrm{B} \Sigma_{1}$, much less $A_{\alpha}=\mathrm{I} \Sigma_{1}$. 


\section{A Result on End-Extension Models}

Theorem 4.1 Let $\mathbf{K}$ be a Kripke model of $\mathbf{H A}$ such that, whenever $\alpha \leq \beta$ in $K$, $A_{\beta}$ is an end extension of $A_{\alpha}\left(A_{\alpha} \subseteq_{e} A_{\beta}\right.$; such $\mathbf{K}$ are called end-extension models). Then, for each $\alpha$ in $K$, we have $A_{\alpha}=B \Sigma_{1}$.

Proof: We have to show that for each $\alpha \in K$, every $\Sigma_{1}$-formula $\varphi(x, y, \bar{v})$ and all $a, \bar{a} \in A_{\alpha}, \alpha \models \forall x<a \exists y \varphi(x, y, \bar{a}) \rightarrow \exists u \forall x<a \exists y<u \varphi(x, y, \bar{a})$. We may assume without loss of generality that $\varphi$ is in fact $\Delta_{0}$. Note that HA proves each instance of the $\Sigma_{1}$-collection scheme (this is easily seen by inspecting the usual proof that PA $\left.\vdash \mathrm{B} \Sigma_{1}\right)$. Hence $\alpha \Vdash \forall x<a \exists y \varphi(x, y, \bar{a}) \rightarrow \exists u \forall x<a \exists y<u \varphi(x, y, \bar{a})$. Now assume that $\alpha \models \forall x<a \exists y \varphi(x, y, \bar{a})$. We are finished if we can show that $\alpha \Vdash \forall x<a \exists y \varphi(x, y, \bar{a})$, since then, our instance of collection being forced at $\alpha$, $\alpha \Vdash \exists u \forall x<a \exists y<u \varphi(x, y, \bar{a})$; the latter sentence being $\Sigma_{1}$, it immediately follows that $\alpha \models \exists u \forall x<a \exists y<u \varphi(x, y, \bar{a})$.

Now $\alpha \Vdash \forall x<a \exists y \varphi(x, y, \bar{a})$ iff $(\forall \beta \geq \alpha) \beta \models \forall x<a \exists y \varphi(x, y, \bar{a})$, as is easily seen by trivial manipulations of the definition of forcing. By assumption $\alpha \models$ $\forall x<a \exists y \varphi(x, y, \bar{a})$; let $\beta \geq \alpha$ and $b \in A_{\beta}, \beta \models b<a$. We need to show that $\beta \models \exists y \varphi(b, y, \bar{a})$. But since $A_{\alpha} \subseteq_{e} A_{\beta}, b \in A_{\alpha}$ already, so $\alpha \models \exists y \varphi(b, y, \bar{a})$ and thus $\beta \models \exists y \varphi(b, y, \bar{a})$ (since $\Sigma_{1}$-sentences are preserved under end extensions).

Remark 4.2 It is unclear how rich the class of end-extension models of HA is, for example, whether HA is complete with respect to this class.

5 Finite Models and Models of Finite Depth In 5, van Dalen et al. developed their method of pruning.

Definition 5.1 Let $\mathbf{K}=\left(K, \leq,\left(A_{\eta}\right)_{\eta \in K}\right)$ be any Kripke structure. Suppose that $\alpha \in K$ and that $\rho \in L_{\alpha}$ is a sentence such that $\alpha \nVdash \rho$. Let $K^{\rho}:=\{\beta \in K: \beta \geq \alpha \& \beta \nVdash$ $\rho$ \}, let $\leq^{\rho}$ be $\leq$ restricted to $K^{\rho}$ and put $\mathbf{K}^{\rho}=\left(K^{\rho}, \leq^{\rho},\left(A_{\eta}\right)_{\eta \in K^{\rho}}\right)$. Forcing in $\mathbf{K}^{\rho}$ is denoted $\Vdash^{\rho}$.

The following lemmas then easily follow.

Lemma 5.2 (First Pruning Lemma) Let $\mathbf{K}$ be any Kripke structure, $\alpha \in K, \rho \in L_{\alpha}$ a sentence, $\alpha \nVdash \rho$. Then for any $L_{\alpha}$-sentence $\varphi$,

$$
\alpha \Vdash \varphi^{\rho} \text { iff } \alpha \Vdash^{\rho} \varphi .
$$

Lemma 5.3 (Second Pruning Lemma) Let $\mathbf{K}$ be a Kripke structure, $\alpha \in K, \rho \in L_{\alpha}$ a sentence, $\alpha \nVdash \rho$ and $\alpha \Vdash \mathbf{H A}$. Then $\alpha \Vdash^{\rho} \mathbf{H A}$. In particular, if $\mathbf{K} \models \mathbf{H A}$, then $\mathbf{K}^{\rho}=\mathbf{H A}$.

Van Dalen et al. then conclude

Theorem 5.4 Let $\mathbf{K}$ be a Kripke model of $\mathbf{H A}$ such that $K$ is finite. Then $\mathbf{K}$ is locally PA.

We will prove this in a slightly more general form.

Theorem 5.5 Let $\mathbf{K} \models \mathbf{H A}$ be such that for some $n<\omega$, no path in $(K, \leq)$ is longer than $n$. Then $\mathbf{K}$ is locally $\mathbf{P A}$. 
Proof: $\quad$ Let $\alpha \in K$ and suppose that $\alpha \not \models \mathbf{P A}$. Then for some semipositive sentence $\varphi, \mathbf{P A} \vdash \varphi, \alpha \not \models$. Prune $\mathbf{K} n$ times by $\varphi$, obtaining a sequence $\mathbf{K}=\mathbf{K}_{0}, \mathbf{K}_{1}, \ldots, \mathbf{K}_{n}$. $\alpha \in K_{i}$ for all $i \leq n$, since $\varphi$ is semipositive, $\alpha \not \models \varphi$, and thus $\alpha \nVdash_{i} \varphi$. By the Second Pruning Lemma, $\alpha \Vdash_{n}$ HA. But $\alpha$ is a terminal node in $K_{n}$ : every instance of pruning reduces the lengths of paths through $\alpha$ at least by one, since terminal nodes which force HA model PA and hence satisfy and force $\varphi$. But every path through $\alpha$ has a length $\leq n$. Now $\alpha \Vdash_{n} \mathbf{H A}$ and $\alpha$ is terminal in $K_{n}$, which implies $\alpha \models \mathbf{P A}$, a contradiction.

Van Dalen et al. remark that there seems to be no straighforward way to extend their methods to infinite models of HA. As Theorem 5.5 shows, this has to be taken with a grain of salt, since Theorem 5.5does apply to certain infinite models (viz. those of finite depth). Together with some new ideas, the methods of 5 can also be used to treat certain Kripke models of infinite depth, in particular those over the frame $(\omega, \leq)$.

6 Models over the frame $(\omega, \leq) \quad$ Van Dalen et al. in [5] already investigated models $\mathbf{K}=\mathbf{H A}$ for which $(K, \leq)=(\omega, \leq)$ and showed that in such models, for infinitely many $n<\omega, n \models \mathbf{P A}$. Using their methods and Theorem 5.4. we will show that such models are in fact always PA-normal. First we need a lemma.

Lemma 6.1 Let $\mathbf{K}=\left(\omega, \leq,\left(A_{n}\right)_{n<\omega}\right) \models$ HA.

1. For any $m<\omega$ and $\varphi(\bar{x})$ in $L_{m}$ not containing $\exists$,

$$
m \Vdash \forall \bar{x}(\varphi(\bar{x}) \vee \neg \varphi(\bar{x})) .
$$

2. If $m \Vdash \psi \vee \neg \psi$ for each $L_{m}$-sentence $\psi$, then for each $\bar{a} \in A_{m}$ and $\psi(\bar{x}) \in L$ not containing $\exists$,

$$
m \Vdash \psi(\bar{a}) \Longleftrightarrow m \models \psi(\bar{a}) .
$$

Proof: The first claim is proved by induction on $\varphi(\bar{x})$. The interesting cases are those of implication and universal quantification. So suppose first that $\varphi \equiv \psi \rightarrow \chi$. We have to show that $m \Vdash \forall \bar{x}[(\psi(\bar{x}) \rightarrow \chi(\bar{x})) \vee \neg(\psi(\bar{x}) \rightarrow \chi(\bar{x}))]$. So let $k \geq m$ and $\bar{c} \in A_{k}$. If $k \Vdash \psi(\bar{c}) \rightarrow \chi(\bar{c})$, we have nothing to show. Suppose $k \nVdash \psi(\bar{c}) \rightarrow \chi(\bar{c})$. Clearly then $k \nVdash \chi(\bar{c})$, and thus by the induction hypothesis $k \Vdash \neg \chi(\bar{c})$. Also $k \Vdash$ $\psi(\bar{c})$ (otherwise by the inductive hypothesis $k \Vdash \neg \psi(\bar{c})$ and thus $k \Vdash \psi(\bar{c}) \rightarrow \chi(\bar{c})$ ). Hence $k \Vdash \neg(\psi(\bar{c}) \rightarrow \chi(\bar{c}))$.

Now suppose that $\varphi(\bar{x}) \equiv \forall y \psi(y, \bar{x})$. We have to show that $m \Vdash \forall \bar{x}(\forall y \psi(y, \bar{x}) \vee$ $\neg \forall y \psi(y, \bar{x}))$. Let $k \geq m$ and $\bar{c} \in A_{k}$. Again, if $k \Vdash \forall y \psi(y, \bar{c})$, there is nothing to show. Otherwise, for some $l \geq k$ and $a \in A_{l}, l \nVdash \psi(a, \bar{c})$. By the induction hypothesis, $l \Vdash \neg \psi(a, \bar{c})$ and hence also $l \Vdash \neg \forall y \psi(y, \bar{c})$. But then $k \Vdash \neg \forall y \psi(y, \bar{c})$.

The second claim is proved by induction on the complexity of $\varphi$. Again, implication and universal quantification are the important cases. Suppose first that $\varphi \equiv$ $\psi \rightarrow \chi$. If $m \Vdash \psi \rightarrow \chi$, in particular $m \Vdash \psi$ implies $m \Vdash \chi$. By induction hypothesis, this entails that $m \models \psi$ implies $m \models \chi$, which is what we need. If $m \nVdash \psi \rightarrow \chi$, then clearly $m \nVdash \chi$ and by the induction hypothesis $m \forall \neq \chi$. Also $m \nVdash \neg \psi$ because otherwise $m \Vdash \psi \rightarrow \chi$. But since by assumption for claim 2, $m \Vdash \psi \vee \neg \psi, m \Vdash \psi$ and by the induction hypothesis again $m \models \psi$. Thus $m \not \models \psi \rightarrow \chi$. 
Now let $\varphi \equiv \forall x \psi(x)$. The $\Rightarrow$ direction is again easy. Suppose that $m \nVdash \forall x \psi(x)$. Then for some $k \geq m$ and $c \in A_{k}, k \nVdash \psi(c)$. By claim 1 (note that $\psi$ is $\exists$-free), $k \Vdash \neg \psi(c)$ and thus $k \Vdash \exists x \neg \psi(x)$. By assumption $m \Vdash \exists x \neg \psi(x) \vee \neg \exists x \neg \psi(x)$, and hence $m \Vdash \exists x \neg \psi(x)$. So for some $a \in A_{m}, m \Vdash \neg \psi(a)$, so $m \nVdash \psi(a)$ and by induction hypothesis, $m \not \models \psi(a)$. Hence $m \not \models \forall x \psi(x)$.

Theorem 6.2 Let $\mathbf{K}=\left(\omega, \leq,\left(A_{n}\right)_{n<\omega}\right) \models$ HA. Then for each $n<\omega, n \models$ PA.

Proof: First suppose that for some sentence $\rho \in L_{n}, n \nVdash \rho$ and for some $m>n$, $m \Vdash \rho$. Then, by definition of $\mathbf{K}^{\rho}, n \in K^{\rho}$. By the Second Pruning Lemma, $\mathbf{K}^{\rho}=\mathbf{H A}$. Moreover, if $m \Vdash \rho$, then for every $r>m, r \Vdash \rho$, and so $\mathbf{K}^{\rho}$ is finite. By Theorem 5.4. $n \models \mathbf{P A}$.

So suppose that for every sentence $\rho \in L_{n}$, if $n \nVdash \rho$, then for all $m>n, m \nVdash \rho$ and thus, by definition of forcing, $n \Vdash \neg \rho$. It follows that for every sentence $\rho \in L_{n}$, $n \Vdash \rho \vee \neg \rho$. By Lemma 6.1. part 2, forcing and classical validity at $n$ coincide for $\exists$-free $L_{n}$-sentences, and in particular, for all $\exists$-free $L$-sentences. Now we must check that every instance of the induction schema is classically valid at $n$. Take any formula $\varphi(x, \bar{y})$. Without loss of generality, we may suppose that $\varphi(x, \bar{y})$ is composed of $\wedge, \rightarrow, \perp$ and $\forall$ only, since we are dealing with classical truth here. Clearly $n \Vdash \forall \bar{y}(\varphi(0, \bar{y}) \wedge \forall x(\varphi(x, \bar{y}) \rightarrow \varphi(S x, \bar{y})) \rightarrow \forall x \varphi(x, \bar{y}))$ since $n \Vdash$ HA. This last sentence is clearly $\exists$-free, and so, as pointed out, by Lemma 6.1 part 2, it is true in the structure $A_{n}$, which had to be shown.

Although slightly off the topic, we note in passing the following characterization of Markov's principle $M_{P R}$ for models over $\omega$.

Theorem 6.3 Let $\mathbf{K}=\left(\omega, \leq,\left(A_{n}\right)_{n<\omega}\right) \models$ HA. The following are equivalent.

1. $\mathbf{K} \models M_{P R}$.

2. For each $n \in \omega$, every $\varphi(\bar{x}) \in \Pi_{1}$ and all $\bar{a} \in A_{n}$,

$$
n \Vdash \varphi(\bar{a}) \Longleftrightarrow n \models \varphi(\bar{a}) .
$$

3. For all $n, m \in \omega$, if $n \leq m$, then $A_{n} \prec_{\Sigma_{1}} A_{m}$.

Proof: $\quad(2) \Rightarrow(3)$ and (3) $\Rightarrow(1)$ were shown in 3 . It remains to prove (1) $\Rightarrow$ (2). The left-to-right direction is clear, so assume that $n \nVdash \forall \bar{x} \psi(\bar{x}, \bar{a})$ with $\psi \in \Delta_{0}$ and $\bar{a} \in A_{n}$. Suppose first that $n \Vdash \neg \neg \exists \bar{x} \neg \psi(\bar{x}, \bar{a})$. By $M_{P R}, n \Vdash \exists \bar{x} \neg \psi(\bar{x}, \bar{a})$ and thus $n \models \exists \bar{x} \neg \psi(\bar{x}, \bar{a})$, i.e., $n \nvdash \forall \bar{x} \psi(\bar{x}, \bar{a})$. Now suppose that $n \nVdash \neg \neg \exists \bar{x} \neg \psi(\bar{x}, \bar{a})$. Then, for some $m \geq n, m \Vdash \neg \exists \bar{x} \neg \psi(\bar{x}, \bar{a})$. Using intuitionistic logic, this entails $m \Vdash \forall \bar{x} \neg \neg \psi(\bar{x}, \bar{a})$, which by decidability of $\Delta_{0}$-formulas implies $m \Vdash \forall \bar{x} \psi(\bar{x}, \bar{a})$. On the other hand, since $n \nVdash \forall \bar{x} \psi(\bar{x}, \bar{a})$, for some $k \geq n$ and some $\bar{c} \in A_{k}, k \nVdash \psi(\bar{c}, \bar{a})$, so $k \Vdash \neg \psi(\bar{c}, \bar{a})$ and thus $k \Vdash \exists \bar{x} \neg \psi(\bar{x}, \bar{a})$. But then $\max (k, m) \Vdash(\exists \bar{x} \neg \psi(\bar{x}, \bar{a})) \wedge$ $(\forall \bar{x} \psi(\bar{x}, \bar{a}))$, a contradiction.

7 Models over frames of other order types Theorems 5.4 and 6.2 together give rise to a number of other order types of frames Kripke models of HA over which are PA-normal. We mention a few examples. 
Example 7.1 (The Comb) $\quad$ Suppose $\mathbf{K} \models \mathbf{H A}$ and $(K, \leq)$ has the following form.

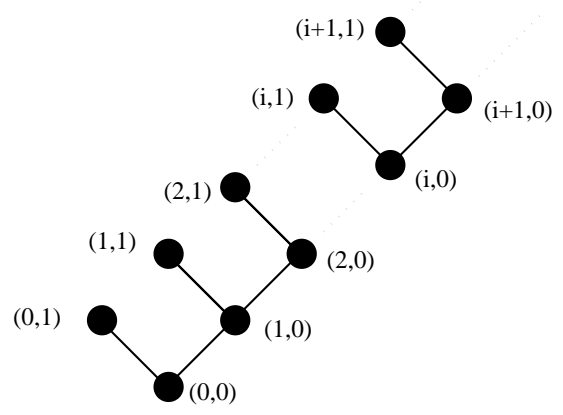

Figure 1: The Comb

Clearly every terminal node $(i, 1)$ is Peano. Suppose $(0,0) \not \models \mathbf{P A}$. Again, for some semipositive theorem $\rho$ of PA, $(0,0) \not \models \rho$ and hence $(0,0) \nVdash \rho$. Now consider $\mathbf{K}^{\rho}$. Since all terminal nodes are pruned away, it is either finite or of order type $\omega$, and $(0,0) \in K^{\rho}$. So either by Theorem 5.4 by theorem 6.2. $(0,0) \models \mathbf{P A}$, a contradiction.

Example 7.2 (The Tick) $\quad$ Let $\mathbf{K} \models \mathbf{H A}$ and $(K, \leq):=$
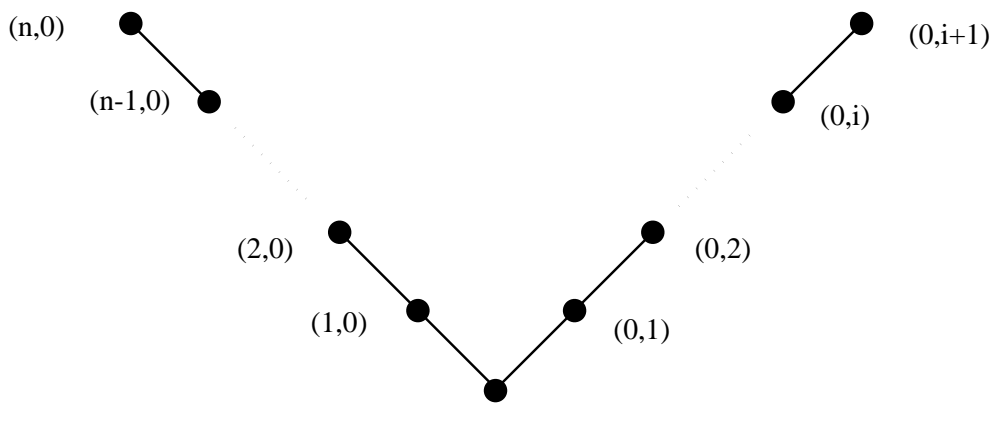

$(0,0)$

Figure 2: The Tick

Again, if $(0,0) \forall \mathbf{P A}$, by at most $n$ prunings we can obtain a model of HA containing $(0,0)$ which is either finite or has order type $\omega$. Hence $(0,0) \models \mathbf{P A}$, a contradiction.

Example 7.3 (V.) $\quad$ Let $\mathbf{K} \models \mathbf{H A}$ and $(K, \leq)$ as in figure 3 .

Suppose $(0,0) \not \mathbf{P A}$. Let $\rho$ be any sentence with $(0,0) \nVdash \rho$ and $\beta \Vdash \rho$ for some $\beta>(0,0) . \mathbf{K}^{\rho}$ is then finite or of the type in Example 7.2 or has order type $\omega$, and in any case contains $(0,0)$, a contradiction. So if $(0,0) \nVdash \rho,(0,0) \Vdash \neg \rho$. By adapting Lemma 6.1 to the present situation, we obtain $(0,0) \models \mathbf{P A}$ as in the proof of Theorem 6.2. a contradiction. 

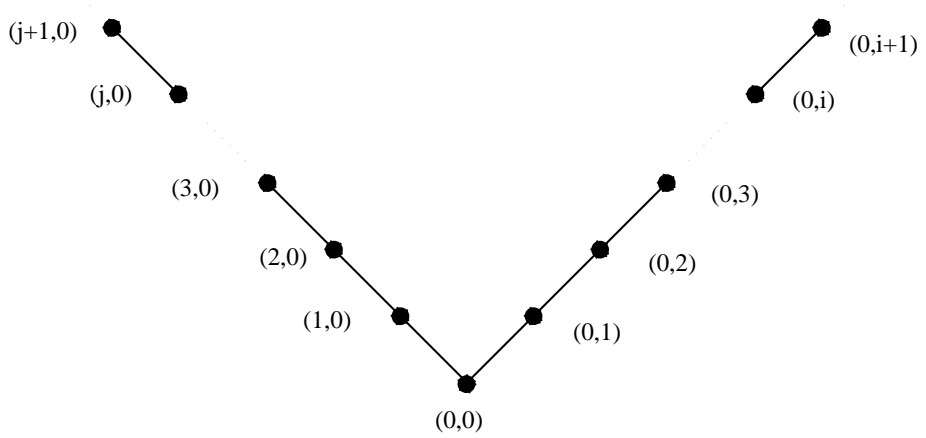

Figure 3: V.

8 A Completeness Theorem The following result, implicit in [1], is in a way the most convincing evidence for our initial conjecture (that Kripke models of HA are PA-normal).

Theorem 8.1 HA is complete with respect to the class $\{\mathbf{K}=\mathbf{H A}: \mathbf{K}$ is locally $\mathbf{P A}\}$.

Proof: In 11, Buss defined a subtheory $\mathcal{H} \mathbf{P A}$ of HA for which he showed the following saturation lemma:

Let $C_{0}$ be a countable set of new constants and $\Gamma$ a set of $L\left(C_{0}\right)$-sentences such that $\mathcal{H} \mathbf{P A} \subseteq \Gamma$. Further suppose $\Gamma \nvdash \varphi$, where $\varphi$ is some $L\left(C_{0}\right)$-sentence. Then for any countable set $C_{1}$ of new constants there is a set $\Gamma^{*}$ of $L\left(C_{0} \cup C_{1}\right)$-sentences such that

1. $\Gamma \subseteq \Gamma^{*}$,

2. $\Gamma^{*}$ is saturated,

3. $\Gamma^{*} \nvdash \varphi$,

4. the term model determined by $\Gamma^{*}$ classically satisfies PA.

Since $\mathcal{H} \mathbf{P A}$ is a subtheory of $\mathbf{H A}$, we may substitute $\mathbf{H A}$ for $\mathcal{H} \mathbf{P A}$ in the lemma and obtain in the standard way a canonical PA-normal model of HA.

This Completeness Theorem implies that in constructing Kripke models of HA, we never need to use classical structures which do not satisfy PA. In the next section we will show directly that in HA-models over converse wellfounded frames, we may simply omit all (if any) non-Peano nodes and obtain a Kripke model of HA in which precisely the same sentences are forced as in the original model.

9 Some General Considerations $\quad$ Let $\mathbf{K} \models \mathbf{H A}$ be arbitrary and $\alpha_{0} \in K, \alpha_{0} \not \models \mathbf{P A}$. It is obvious that $\alpha_{0}$ cannot become a terminal node by an application of pruning (and in fact, by Theorem 5.4, whenever $\alpha_{0} \nVdash \rho$, there are infinitely many $\beta>\alpha_{0}$ such that $\beta \nVdash \rho$ ). We thus obtain for any sentence $\rho \in L_{\alpha_{0}}$

$$
\text { (*) } \quad \alpha_{0} \Vdash \rho \Longleftrightarrow \forall \beta>\alpha_{0}: \beta \Vdash \rho .
$$

This situation may be described by saying that $\alpha_{0}$ is a redundant node in $\mathbf{K}$, for it encodes no information not already present above it. The following proposition makes this more precise. 
Proposition 9.1 Let $\mathbf{K}$ and $\alpha_{0}$ be as above. Let $\mathbf{K}^{-}=\left(K^{-}, \leq^{-},\left(A_{\alpha}\right)_{\alpha \in K^{-}}\right)$, where $K^{-}=K-\left\{\alpha_{0}\right\}$, $\leq^{-}$is $\leq$restricted to $K^{-}$. Forcing in $\mathbf{K}^{-}$is $\Vdash^{-}$. Then for all $\beta \in K^{-}$and every $L_{\beta}$-sentence $\rho$,

$$
\beta \Vdash \rho \Longleftrightarrow \beta \Vdash^{-} \rho .
$$

In particular, for every L-sentence $\varphi$,

$$
\mathbf{K} \Vdash \varphi \Longleftrightarrow \mathbf{K}^{-} \Vdash^{-} \varphi .
$$

Proof: By induction on $\rho$, simultaneously for all $\beta \in K^{-}$. The atomic case is trivial, as are (using the induction hypothesis) the cases of conjunction, disjunction and existential quantification. Let us check the case of implication.

First suppose $\beta \Vdash \psi \rightarrow \chi$, i.e., for all $\gamma \geq \beta$, if $\gamma \Vdash \psi$, then $\gamma \Vdash \chi$. In particular, for all $\gamma \geq^{-} \beta, \gamma \Vdash \psi \Rightarrow \gamma \Vdash \chi$. By the induction hypothesis, for all $\gamma \geq^{-} \beta, \gamma \Vdash^{-}$ $\psi \Rightarrow \gamma \Vdash^{-} \chi$, i.e., $\beta \Vdash^{-} \psi \rightarrow \chi$.

Now suppose that $\beta \nVdash \psi \rightarrow \chi$, i.e., for some $\gamma \geq \beta, \gamma \Vdash \psi$ and $\gamma \nVdash \chi$.If $\gamma$ is not $\alpha_{0}$, we are finished by the induction hypothesis. If $\gamma$ is $\alpha_{0}$, by (*) there is some $\delta>\alpha_{0}, \delta \nVdash \chi$ (and of course $\left.\delta \Vdash \psi\right)$; this $\delta$ is in $K^{-}$, so again we are finished by the induction hypothesis. The case of universal quantification is treated analogously.

In the case in which $(K, \leq)$ is conversely well founded we may in fact remove all non-Peano nodes at once without changing the theory of the model.

Proposition 9.2 Let $\mathbf{K}=\mathbf{H A}$ be such that $(K, \leq)$ is conversely well founded (that is, there are no infinite strictly ascending chains). Put $K^{-}=\{\alpha \in K: \alpha \models \mathbf{P A}\}$ and let $\leq^{-}$be $\leq$restricted to $K^{-}$. Then $\mathbf{K}^{-}=\left(K^{-}, \leq^{-},\left(A_{\alpha}\right)_{\alpha \in K^{-}}\right) \models \mathbf{H A}$ and in fact, for $\alpha \in K^{-}$and $\rho$ an $L_{\alpha}$-sentence,

$$
\alpha \Vdash \rho \Longleftrightarrow \alpha \Vdash^{-} \rho .
$$

Proof: First note that $K^{-} \neq \varnothing$ since $\mathbf{K}$ contains terminal nodes. We proceed by induction on $\rho$. The atomic case is clear by definition, and the cases of conjunction, disjunction and existential quantification are easy, using the induction hypothesis.

Consider the case where $\rho$ is $\psi \rightarrow \chi$. The left-to-right direction is again easy, so suppose that $\alpha \nVdash \psi \rightarrow \chi$, i.e., for some $\beta_{0} \geq \alpha, \beta_{0} \Vdash \psi$ and $\beta_{0} \nVdash \chi$. If there is such a $\beta_{0}$ in $K^{-}$we are finished by the induction hypothesis. Suppose the only such $\beta_{0}$ are in $K-K^{-}$. Since $\beta_{0} \not \models \mathbf{P A}, \beta_{0}$ is redundant in the sense of $(*)$ and thus there is some $\beta_{1}>\beta_{0}$ such that $\beta_{1} \Vdash \psi, \beta_{1} \nVdash \chi$. By assumption, $\beta_{1} \not \models \mathbf{P A}$, so there is a $\beta_{2}>\beta_{1}$ with $\beta_{2} \Vdash \psi, \beta_{2} \nVdash \chi$. Continuing in this way, we can construct an infinite strictly ascending chain $\beta_{0}<\beta_{1}<\beta_{2}<\ldots$, contradicting the fact that $(K, \leq)$ is conversely well founded. The case of universal quantification is entirely analogous.

Remark 9.3 Note that in Propositions 9.1 and 9.2 even if $\mathbf{K}$ is rooted, $\mathbf{K}^{-}$need not be, since the bottom node of $\mathbf{K}$ may have been deleted (for not being Peano). We may however tag the standard model $\mathbb{N}$ below all the nodes of $\mathbf{K}^{-}$(cf. [4]) to obtain a new, rooted HA-model $\left(\mathbf{K}^{-}\right)^{\prime}$ for which we have for all L-sentences $\varphi$, by Proposition 9.1 ,

$$
\mathbf{K} \nVdash \varphi \Rightarrow\left(\mathbf{K}^{-}\right)^{\prime} \nVdash \varphi \text {. }
$$


Remark 9.4 Proposition 9.2 is of course in accord with Theorem 5.4. completeness of HA with respect to PA-normal models. One might expect that the set of nonPeano nodes could be safely omitted from arbitrary models of HA without altering the theory of the model, that is, that Proposition 9.2 remains true even when the hypothesis of converse wellfoundedness is removed. This would follow immediately if it could be shown that every Kripke model of HA contains at least one Peano node (sketch of proof: proceed as in the proof of Proposition 2.2. we need only to modify the proofs of the right-to-left direction of the implication and universal quantification cases. Consider for example, implication. Suppose $\alpha \Vdash^{-} \psi \rightarrow \chi$, i.e., for all $\gamma \geq \alpha$, if $\gamma \models \mathbf{P A}$ and $\gamma \Vdash \psi$, then $\gamma \Vdash \chi$. Let $\beta \geq \alpha$ and $\beta \Vdash \psi$. If $\beta \nVdash \chi$, consider $\mathbf{K}_{\beta}^{\chi}$. By the Second Pruning Lemma, $\mathbf{K}_{\beta}^{\chi} \models \mathbf{H A}$. But all Peano nodes have been pruned away. So, $\mathbf{K}_{\beta}^{\chi}$ would be an HA-model containing no Peano node, a contradiction.). By Theorem 8.1 it is certainly true that there are Peano nodes arbitrarily high up the strong canonical model for HA.

Acknowledgments I wish to thank Albert Visser, Dick de Jongh, and my thesis supervisor, Professor Diller, for reading and commenting on preliminary versions of this paper and for their interest in and support of my work. I am very grateful to an anonymous referee who pointed out a serious error in the first version of the article.

\section{REFERENCES}

[1] Buss, S., "Intuitionistic Validity in T-normal Kripke Structures," Annals of Pure and Applied Logic, vol. 59 (1993), pp. 59-173. Zbl 0802.03006 MR 94m:03020 1.12.8.18

[2] Kaye, R., "Using Herbrand-type theorems to separate strong fragments of arithmetic," pp. 238-245 in Arithmetic, Proof Theory, and Computational Complexity, edited by P. Clote and J. Krajicek, Clarendon Press, Oxford, 1993.Zbl 0803.03038 MR 94f:03067 3.2

[3] Markovic, Z., "On the Structure of Kripke Models of Heyting Arithmetic," Mathematical Logic Quarterly, vol. 39 (1993), pp. 531-538.Zbl 0805.03050|MR 95f:03103 1. $1,3,6$

[4] Smoryński, C., "Applications of Kripke Models," pp. 324-391 in Metamathematical Investigations of Intuitionistic Arithmetic and Analysis, edited by A. S. Troelstra, Springer-Verlag, Berlin, 1973.Zbl 0275.02025||MR 48:3699||MR 56:8334 1, 9.3

[5] Van Dalen, D., H. Mulder, E. C. W. Krabbe, and A. Visser, "Finite Kripke Models of HA are Locally PA," Notre Dame Journal of Formal Logic, vol. 27 (1986), pp. 528-532. Zbl 0632.03048 |MR 87m:03088 1.5.5.6

Institut für Mathematische Logik und Grundlagenforschung

Einsteinstraße 62

Westfälische Wilhelms-Universität Münster

D-48149 Münster

GERMANY

email: Kai.Wehmeier@math.uni-muenster.de 